\title{
Efectos de facilitación e interferencia en el procesamiento de información
}

\author{
JAVIER SÁINZ, JUAN MAYOR y JAVIER GONZÁLEZ-MARQUÉS \\ Departamento de Procesos Cognitivos, Universidad Complutense
}

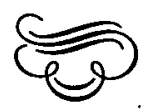

\section{Resumen}

En un análisis de la representación debe distinguirse entre el conocimiento registrado a nivel de la memoria semántica y su uso y manipulación a nivel de la memoria activa. La diferenciaciōn funcional de estos niveles es posible si se especifican los sistemas y mecanismos que intervienen en el procesamiento de información. Los efectos de facilitación e interferencia comünmente hallados en los paradigmas experimentales de anticipación y Stroop permiten describir algunos de los sistemas y mecanismos que subyacen a las imágenes mentales y a las palabras como distintos tipos de representación simbólica. Frente a una teoria interpretativa, una teoría constructiva explica las propiedades emergentes de las imágenes mentales como el resultado de la actualización de su referencia en un determinado contexto. Distinguiendo entre conocimiento registrado y uso del conoci. miento es posible rechazar la perspectiva de Pylyshyn en el análisis de las imágenes mentales. En este artículo, a modo de introducción, presentamos algunos de los trabajos que estudian la base funcional de los efectos de facilitación e interferencia. Estos efectos, que también se especifican, se han venido utilizando para caracterizar los mecanismos que intervienen en el procesamiento de

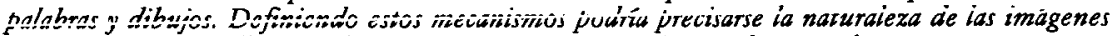
mentales y cómo se lleva a cabo su interpretación semántica. En los artículos que se presentan se pone de manifiesto que el distinto procesamiento estratégico a que se someten dibujos y palabras depende del proceso de decisión y éste a su vez de las demandas de la tarea.

\section{Abstract}

In order to study mental representations, a functional distinction must be made between the knowledge stored at the semantic memory level and its use and manipulation at the working memory level. This functional distinction can be made if the information processing mechanism and systems involve are specified. The facilitation and interference effects, often found in the stroop and priming paradigms, allow for the descriptions of those mechanisms underlying mental images and words as different simbolyc representations. Contrary to an interpretative theory, a constructive theory explains the emergent properties of mental images as deriving from the actualization of the reference provided by the context. Distinguishing between stored knowledge and use of knowledge forces the rejection of Pylyshyn's approaches to mental image analysis. Specifically, in this article as a way of introduction, papers studying the functional basis of the facilitation and interference effects are presented. This effects empirically specified, are used to characterize the word-and picture processing mecanisms. The mechanisms might clarify how the semantic interpretation of mental images is made. In the present articles it is showed that the difference nature of strategic proccesing of pictures and words depends on decision processes as activated from tasks demands.

Dirección del autor: Javier Sainz. Departamento de Psicología Básica: Procesos cognitivos. Facultad de Psicología. Universidad Complutense de Madrid. Somosaguas. 28023 Madrid. 
El interés de la crítica de Pylyshyn $(1981,1984)$ a la noción de imagen mental no deriva de que niegue su papel causal en el comportamiento sino en que este papel lo atribuya al conocimiento implícito a partir del que se forma e interpreta, de acuerdo con algún tipo de función de composición e interpretación semántica. En efecto, de la atribución de un papel causal a las imágenes mentales no se infiere que sus propiedades no deriven de la base de datos del sistema, ni tampoco que sean, en sentido estricto, emergentes. La noción de imagen mental, diferente para Kosslyn (1980), y Pylyshyn (1984), a pesar de su común referencia al paradigma computacional, y Paivio (1986), no puede explicarse sin distinguir entre su función y las propiedades que la caracterizan como representación. Aunque rechaza la naturaleza primitiva de las imágenes mentales al interpretarlas en términos sintácticos, - argumento que resta a la crítica de Pylyshyn (1984) parte de su sentido-, Kosslyn (1980, p. 25) defiende el papel causal de las imágenes mentales en el comportamiento y el carácter emergente de sus propiedades «que no se derivan necesariamente de la constitución molecular de los objetos implicados».

Una imagen mental puede definirse, de acuerdo con Kosslyn (1980), como un tipo de representación de naturaleza no primitiva, analógica y directa, en la que «las propiedades de sus partes y sus relaciones mutuas en la configuración en que se expresa representan propiedades y relaciones de una configuración representada compleja, de tal forma que la estructura de la representación proporciona cierta información acerca de la estructura de lo que representas (Sloman, 1971, p. 213). El concepto de analogía no identifica, en sentido estricto, una clase de representaciones sino una serie de propiedades de una función interpretativa que verifica la existencia de una relación funcional (Pylyshyn, 1975, 1978), homomórfica (Hayes, 1974; Shepard, 1975), entre la forma lógica de una representación y la forma lógica del dominio que representa, dada una descripción independiente.

La condición que ha de cumplir una representación para ser directa es que su interpretación no derive de la composición de una serie de componentes semánticos primitivos sino que resulte de una aplicación entre una descripción independiente del dominio que representa y la descripción que proporciona como representación. Un sistema cognitivo que opere en tiempo real obtiene una descripción independiente de sus representaciones o creencias a través del análisis perceptivo y el análisis de su propia actividad y de los efectos inducidos por ésta, de acuerdo con el principio de racionalidad (Newell, 1982; Pylyshyn, 1984). Al definirla en estos términos, una imagen mental requiere la adopción de una teoría semántica para explicar sus propiedades. Su función en el comportamiento depende así de cómo se caractericen los procesos que concluyen en su interpretación.

\section{TEORIAS INTERPRETATIVAS VERSUS TEORIAS CONSTRUCTIVAS}

Respecto de la interpretación semántica de una imagen mental, puede adoptarse o bien una teoría semántica interpretativa o bien una teoría semántica constructiva. En una teoría semántica interpretativa, la interpretación de una imagen mental se deduce, en sentido estricto, de la composición de una serie de componentes primitivos según especifica una cierta función, contextualmente independiente, que es posible definir $a$ priori (cf. Pylyshyn, 1981, 1984). En una teoría semántica constructiva, la interpretación de una representación deriva de la composición de sus primitivos y de las relaciones funcionales que se establecen entre ésta y una serie de descripciones independientes, cognitivamente impenetrables, según una función de composición e interpretación, contextualmente dependiente ( $\mathrm{cf}$. Kosslyn y Schwartz, 1977; Kosslyn, 1980), que es posible definir empíricamente (cf. Anderson, 1978). Una teoría semántica interpretativa sólo reconoce relaciones analógicas a nivel de la base de datos del sistema. Una teoría semántica constructiva reconoce que dichas relaciones analógicas pueden darse, de hecho, en distintos niveles de representación. En tanto una teoría constructiva supone la interacciónl del conocimiento implícito en la base de datos del sistema y el contexto que activa la información que contiene, una teoría interpretativa supone la independencia funcional del conocimiento implícito y el contexto, atribuyendo en exclusiva a aquél el papel que en la conducta cumple una imagen mental.

Un corolario de la crítica de Pylyshyn (1984) al concepto de imagen mental es la distinción entre conocimiento implícito y conocimiento explícito, entre el conocimiento registrado y su manipulación y uso. Se trata de determinar si las propiedades del conoci- 
miento que incorpora una imagen mental proceden, en sentido estricto, de la base de datos que la informa, o presenta, por el contrario, propiedades que no se deducen de las que caracterizan aquella.

Pylyshyn (1984) define el conocimiento implícito como un tipo de conocimiento no accesible libremente. Sus datos, explica Pylyshyn, «no pueden formar parte como premisa de ningún tipo de interferencia válida a menos que se proporcionen los indicadores de recuperación pertinentes» (cf. Williams y Hollan, 1981), que dependen ade cómo se halla estructurado el conocimiento, lo que depende a su vez de cómo, en primer término, se haya adquirido, qué indicadores y qué secuencia es la mejon (Pylyshyn, 1984, p. 247). A menos que se suponga que dependen del contexto generado por el propio sistema, la activación de conocimiento implícito no procede sino del procesamiento de información en tiempo real.

Un sistema computacionalmente cerrado que dependa, para activar su conocimiento, de su propio contexto, y no del procesamiento de información en tiempo teal, es incapaz de alterar sus estados con independencia de algún tipo de funciones preespecificadas, o, en otros términos, es incapaz de aprender. Por otra parte, la tesis de que el conocimiento implícito se activa en función de la información que se procesa ha sido ampliamente demostrada al distinguir entre reconocimiento y recuerdo (cf. Gillund y Shiffrin, 1981; Jacoby, 1983; Johnston, Dark y Jacoby, 1985; Tulving, 1981, 1982). El conocimiento implícito adquiere en la imagen mental una determinación, en función del contexto, de la que carece su base de datos. Los datos de que se compone son de naturaleza analítica y mantienen entre sí relaciones que es posible especificar en términos proposicionales (cf. Kosslyn, 1980).

\section{INTENSION Y EXTENSION}

De acuerdo con Pylyshyn (1984́, p. 230), quienes defienden el carácter emergente de las propiedades que caracterizan una imagen mental son presos de una ilusión semántica: tienden a aver los procesos cognitivos en términos de las propiedades de los objetos que representan -es decir, en términos de la semántica de la representación- más que de la estructura, o sintaxis de la representación», a pesar de que se! mecañismó cữno tai no tiene ningún acceso a las propiedades del dominio representado excepto en cuanto se codifican en la representación mismas. El sistema cognitivo carece, en efecto, de procedimientos que le permitan determinar el valor de verdad de una representación independientemente del dominio que representa, en el contexto en que se actualiza como expresión. La extensión de una expresión formal sólo puede tratarse en términos funcionales de acuerdo con una descripción independiente del dominio representado. Así «un principio de procesamiento mental debe establecerse en términos de las propiedades estructurales formales de sus representaciones, no lo que se supone que en teoría representan.»

Sin embargo, que el sistema cognitivo carezca a priori de procedimientos efectivos para determinar la extensión de una representación no implica necesariamente que su interpretación semántica proceda, en sentido estricto, del conocimiento previo o registrado. Por tanto, las propiedades que caracterizan la interpretación semántica, de una imagen mental no han de proceder necesariamente de la misma base, como un mero epifenómeno de la combinación sintáctica de los datos que integra, sino de la relación funcional de esta representación con algún tipo de descripciones independientes, especificables, que activan aquella. La interacción entre percepción e imaginación justifica, frente a Pylyshyn (1984), la tesis que explica las propiedades emergentes de una imagen mental en términos de una aplicación formal entre descripciones (cf. Farah, 1985).

Una semántica interpretativa, del tipo de la que propone Pylyshyn (1984) para justificar el papel de las imágenes mentales en el comportamiento, no carece de problemas, en rigor insuperables. La teoría no presenta sino explicaciones circulares al reducir la interpretación semántica de una imagen mental a la intensión de sus términos y relaciones. Esta circularidad se expresa en: a) la ausencia de restricciones empíricas acerca de las imágenes mentales que pueden teóricamente formarse (Kosslyn, 1981); b) la falacia simbólica que supone tratar la interpretación semántica de una imagen mental en términos sintácticos; y c) la ineptitud de la teoría para justificar la extensión de una imagen mental como representación. La cuestión crítica es como establecer una relación entre el sentido de una expresión formal y su referencia, siendo que ésta no puede caracteri- 


\section{2}

zarse como una propiedad estrictamente sintáctica y que el sistema carece, por tanto, de procedimientos efectivos para determinarla a priori. Pueden reconocerse las dificultades empíricas de una teoría semántica interpretativa en aquellos casos donde la interpretación semántica de una imagen mental requiere recurrir al contexto que representa (cf. Segalowitz, 1982). Las imágenes mentales deben tratarse como un tipo de representaciones que forman parte de modelos mentales capaces de integrar un tipo distinto de información proposicional.

«Los modelos mentales son -explican Rouse y Morris (1986, p. 351)- mecanismos a través de los cuales los seres humanos son capaces de generar descripciones de los propósitos del sistema y formar explicaciones del sistema en su funcionamiento, de sus estados así como predicciones de sus estados futuros. Es importante subrayar que esta definición no diferencia entre el conocimiento que es simplemente recuperado y el conocimiento que implica algún tipo de cálculo. De este modo, los modelso mentales no son necesariamente modelos computacionales», al menos, no en el sentido de concebirse como representaciones cuya interpretación puede caracterizarse independientemente de algún tipo de descripciones del contexto cognitivamente impenetrables.

Una relación que se deduce a partir de otras especificadas en la base de datos no presenta propiedades emergentes respecto de las que caracterizan el conocimiento que dicha base contiene. Sin embargo, un sistema que actúe de acuerdo sólo a partir de la configuración formal de las expresiones que definen su base de datos carece de procedimientos efectivos que permitan adecuar sus representaciones a las precisas en el comportamiento (cf. Moore, 1975; Rieger, 1975). Dado que una base de datos permite la construcción de un número indefinido de proposiciones, sólo la restricción de este número ya presenta propiedades emergentes respecto del conjunto que es posible formalmente caracterizar. El conocimiento implícito podría reducirse, por definición, a las propiedades directamente especificadas en la base de datos si no fuera porque las relaciones explícitas en dicha base pueden ser un efecto emergente de su activación ante indicadores apropiados del medio. La noción de conocimiento implícito debe abarcar tanto las relaciones efectivamente representadas en la base de datos como las que pueden establecerse por composición de las relaciones explícitas en dicha base. El único límite a esta definición es que el sistema cognitivo carece de un modelo a priori de qué mundos, de entre los posibles, podrían efectivamente caracterizarse a partir del conocimiento de que dispone en su base de datos.

De acuerdo con esta definición, un modelo mental no puede presentar ningún tipo de propiedades emergentes respecto del conocimiento implícito, a menos que incorpore descripciones de un contexto. El contexto determina: a) la activación de la base de datos; b) la inducción de un modelo mental a partir del conocimiento que contiene y la formación de inferencias; y, c) la formación de una descripción de la situación que difiere de la estrictamente representada. El sistema cognitivo es incapaz de generar nuevo conocimiento independientemente de un contexto de representación e independientemente de que el contexto induzca, de hecho, la formación de nuevas descripciones a partir de él.

\section{NIVELES DE REPRESENTACION}

El análisis de las propiedades que caracterizan a las imágenes mentales exige reconocer la existencia de dos distintos niveles de representación. La información que puede representarse en el retén visoespacial de la memoria activa (Baddeley, 1986) se registra de forma no espacial en la memoria semántica o a largo plazo. La memoria semántica incluye información espacial, etiquetada por los mecanismos que acceden a ella como imaginaria, en forma analítica - p. ej. transformadas de Fourier-e información analítica, etiquetada por los mecanismos que acceden a ella como lingüística, - de formato no necesariamente distinto- especificable en términos de proposiciones. El sistema de la memoria activa incluye representaciones relativas a un contexto diferentes según la modalidad y el tratamiento que exige la tarea a realizar. La recuperación automática de la información que contiene la memoria semántica es diferente dependiendo de su modalidad de codificación y el retén de la memoria activa implicado.

La distinción entre dos niveles de representación, definidos por la información registrada en la memoria semántica y por la información que se manipula en los distintos retenes de modalidad de la memoria activa, permite a su vez distinguir entre conocimiento registrado y su uso o manipulación sintáctica en un contexto. La estructura ana- 
lítica de la base de datos en la memoria semántica no supone la codificación y recuperación de un único tipo de información, ni obliga a adoptar un único tipo de representaciones a nivel de la memoria activa, que se encarga tanto de resolver como de adaptar el conocimiento previo a lo que el medio impone en la resolución de un problema. Que se confirme o no la tesis de un formato unitario de codificación (cf. Kosslyn y Pomerantz, 1977; Snodgrass, 1984) no implica necesariamente la existencia de un único nivel de representación; por el contrario, es posible distinguir entre dos niveles de representación según la distinta base funcional de sus respectivas representaciones e, incluso, distintos tipos entre éstas, según la clase de información semántica que contienen y sus relaciones con el dominio que representan.

Dos tipos de representaciones, al menos, pueden distinguirse a nivel de la memoria activa; un tipo de representaciones cuya forma lógica es icomórfica a la del dominio que representa, expresado en términos de una descripción independiente (cf. Kosslyn, 1980), y un tipo de representaciones cuya forma lógica exige la construcción de un modelo mental de su sentido y referencia (cf. Johnson-Laird, 1983: van Dijk y Kintsch, 1983). Los términos de imagen mental y de palabra han sido y son aún, respectivamente, los referentes más clásicos de ambos tipos de representaciones. Por su peculiar estructura sintáctica se han utilizado como estímulos representativos de ambos tipos, dibujos y palabras, bajo el supuesto de que exigen una distinta manipulación de la información codificada en la base de datos, y que estas operaciones son análogas a las que tienen lugar sobre sus respectivas representaciones internas.

\section{LOS EFECTOS DE FACILITACION E INTERFERENCIA EN EL ANALISIS DE LA REPRESENTACION}

De la existencia de dos niveles de representación puede presentarse un número considerable de experiencias y observaciones empíricas que la confirman desde distintas perspectivas teóricas. Sin embargo, y más allá de esta evidencia, la naturaleza de los mecanismos específicos que operan en ambos niveles de representación y su organización funcional no son, todavía, bien conocidos, a pesar de su intrínseca relevancia para el debate que históricamente se ha ocupado de las propiedades de las imágenes mentales y de lâs pálábiàs cumo dos tipos distintos de representación. ¿En qué medida las imágenes mentales representan la información codificada en la base de datos y en qué términos se relacionan con otras formas de representación? Dada la especificidad de las imágenes mentales y, aún de las palabras, debe existir algún tipo de conocimiento cuya independencia del contexto permita una descripción unitaria del mundo que representa independientemente de la contigüidad de los hechos en la experiencia.

Una perspectiva de análisis es la que históricamente ha surgido en torno al procesamiento semántico de los dibujos y de las palabras sirviéndose de los efectos de facilitación e interferencia habitualmente hallados en los paradigmas de anticipación y de Stroop. La integración progresiva de estos paradigmas es un paradigma único, y los efectos asimétricos a menudo observados dependiendo de la modalidad de los estímulos y las demandas de las tareas han permitido identificar dos niveles de representación de cuya actuación paralela son responsables la memoria activa y la memoria semántica. Manipulando la relación semántica entre el estímulo irrelevante y el estímulo objetivo y controlando así los efectos de facilitación e interferencia, es posible conocer algunos de los mecanismos que intervienen en el procesamiento de los dibujos y las palabras e identificar su respectiva base funcional. Graf y Schacter (1987) constatan que en el aprendizaje de nuevas asociaciones, la manipulación del efecto de interferencia afecta sólo a la memoria explícita cuando debería darse a nivel de la memoria implícita si la nueva información se codificara directamente. El carácter estratégico o automático de estos efectos permite especificar qué clase de mecanismos subyacen al procesamiento de estímulos que difieren en su modalidad de presentación.

Glaser (Glaser y Glaser, 1892: Glaser y Düngelholf, 1984) atribuye el efecto de interferencia de Stroop a un proceso de decisión que actúa en el momento en que la memoria activa evalúa «semánticamente la información» (1982, p. 893). «De este modo, el fenómeno de interferencia de Stroop (...) depende de factores estratégicos (y) no puede observarse en los estudios en que se utilizan cuatro o más contingencias de estímulorespuesta (p. ej., estudios en los que existen cuatro o más clases de respuesta) (Logan, Zbrodoff y Williamson, 1984, p. 138). Por el contrario, los efectos de facilitación en un 
paradigma de anticipación serían, en principio, indiscutiblemente automáticos en tanto no se vieran afectados por un proceso de decisión. La Heij, Van der Hejden y Schreuder (1985) suponen que ambos paradigmas reflejan un mecanismo de procesamiento común: las diferencias entre ambos paradigmas pueden reducirse al rango de respuestas que permiten, el número de dominios semánticos y al papel de los estímulos como objetivos o coomo distractores. Igualando el tratamiento experimental de las variables que se manipulan en ambos paradigmas y conservando como única diferencia la secuencia temporal de los distintos componentes de estímulo - condición que es crítica para diferenciar entre un paradigma de anticipación y un paradigma de Stroop-es posible analizar la relevancia de los procesos de decisión en la evaluación semántica del material presentado y distinguir entre dos niveles de representación dependiendo del comportamiento de los efectos de facilitación e interferencia.

En los trabajos de Glaser y Düngelholff (1984), de la Heij, Van der Hejden y Schreunder (1985) y de Mayor, Sáinz, y González-Marqués (1986), cuya traducción se incluye a continuación*, se analizan, en distintos diseños experimentales, los efectos de facilitación e interferencia utilizando los paradigmas de anticipación y de Stroop, con objeto de identificar los mecanismos responsables de estos efectos y la forma en que son modulados por las variables que se manipulan y específicamente la de modalidad. Los resultados experimentales que se obtienen en estos análisis experimentales contribuyen decisivamente a definir el papel de la memoria activa en el procesamiento de información, reduciendo el problema del formato de codificación al tratamiento que este sistema hace de la información para adecuar sus representaciones implícitas a las que exige formar el contexto. Las propiedades emergentes que parecen caracterizar a las imágenes mentales pueden bien proceder de esa relación funcional que se establece entre una representación y una descripción del contexto independiente de aquélla. Que los efectos de facilitación e interferencia afecten de un modo distinto a la memoria activa y a la memoria semántica y que no respondan a un modelo de procesamiento aditivo justifican la existencia de dos niveles de representación y dos tipos de sistemas actuando en paralelo.

\section{Referencias}

ANDERSON, J.R. (1978). Arguments concerning representations for mental imagery. Psychological Review, 85 (4), 249-277.

BADDELEY, A. (1986). Working memory. Oxford series. Oxford: Oxford University Press.

FARAH, M. (1985) Psychophysical evidence for a shared representational medium for mental images and percepts. Journal of Experimental Psychology: General, 114 (1), 91-103.

GILUND, G., y SHIFrRIN, R. (1981). Free recall of complex pictures and abstract words. Joumal of Verbal Learning and Verbal Behavior, 20 (5), 575-592.

GLASER, M.O., y GLASER. W.R. (1982). Time course analysis of the stroop phenomenon. Joumal of Experimental Psychology: Human Perception and Performance, 8, 875-894.

GLASER. W.R., y DUNGELHOFF, F.J. (1984). The time course of picture-word interference. Joumal of Experimental Psychology: Human Perception and Performance, 10 (5), 640-654 (Traducido en este volumen).

GRAF, P., y SCHACTER, D.L. (1987). Selective effects of interference on implicit and explicit memory for new associations. Joumal of Experimental Psychology: Leaming, Memory and Cognition, 13, $1,45-43$.

HAYES. J.P. (1974). Some problems and non-problems in representation theory. British Computer Society, AI and Simulation of Behavior Group Summer Conference. University of Sussex, 63-79.

JACOBY, L.L. (1983). Perceptual enhancement: Persistent effects of an experience. Journal of Experimental Psychology: Leaming, Memory and Cognition, 9 (1), 21-38.

JOHNSON-LAIRD. PH. (1983). Mental models. Towards a cognitive science on language inference and consciousness. Cambridge: Cambridge University Press.

JOHNSTON, W., DARK. V., y JACOBY. L. (1985). Perceptual fluency and recognition judgements. Journal of Experimental Psychology: Learring, Memory and Cognition, 11 (1), 3-11.

KossLYN, S. (1980). Image and mind. Cambridge, Mass: Cambridge University Press.

KossLYN, S. (1981). The medium and the message in mental imagery: A theory. Psychological Review, 88, 46-66.

KOSSLYN, S., y POMERANTZ, J.R. (1977). Imagery, propositions, and the form of internal representations. Cognitive Psychology, 9 (1), 52-76.

* Ia traducción de los artículos que se incluyen es de J. Sáinz. 
Kosslyn. S., y SCHWARTz, S. (1977). A simulation of visual imagery. Cognitive Science, 1 (3), 265-295.

LA HEIJ. W., VAN DER HEIJDEN, A.H.C., y SCHREUdER, R. (1985). Semantic Priming and Stroop-like interference in word-naming tasks. Joumal of Experimental Psychology: Human Perception and Performance, 11 (1), $62-80$ (Traducido en este volumen).

MAYOR, J., SÁINZ, J., y GONZńlez-MARQuÉs, J. (1986). Stroop and priming effects in naming and categorizing tasks using words and pictures. En M. Denis, J. Engelkamp y J. Richardson (eds.): Neuropsychological and cognitive approaches to mental imagery. Amsterdam: Martinus-Nihjoff (Traducido en este volumen).

MOORE, R. (1975). Reasoning from incomplete knowledge in a procedural deductive system. AI Laboratory. Cambridge, Mass: M.IT.

NEWELL, A. (1982). The knowledge level. Artificial intelligence, 18 (1), 87-127.

PYLYSHYN, Z.W. (1975). Do we need images and analogs? Workshop on Theoretical Issues in Natural Language Processing, 1, 174-177.

PYLYSHYN, Z.W. (1978). Imagery and artificial intelligence. En C.W. Savage (ed.): Perception and Cognition: Issues in the Foundations of Psychology. Minnesota Studies in the Philosophy os Science, 9. Minneápolis: University of Minnesota Press.

PYLYSHYN, Z.W. (1981). The imagery debate: Analogue media versus tacit knowledge. Psychological Review, 88 (1), 16-45.

PYIYSHYN, Z.W. (1984). Computation and Cognition. Cambridge: Mass. The MIT Press.

RIEGER, C. (1975). Conceptual memory and inference. En Schank, R.C. Conceptual information processing. Nueva York: North-Holland.

ROUSE, W.B., y MORRIS, N.M. (1986). On looking into the black box: Prospects and limits in the search for mental models. Psychological Bulletin, 100 (3), 349-363.

SEGALOWITZ, N.S. (1982). The perception of semantic relations in pictures. Journal of Experimental Psychology: Memory and Cognition, 10 (4), 381-388.

SHEPARD, R.N. (1975). Form, formation and transformation of internal representations. En R.L. Solso (ed.): Information processing in cognition: The Loyola Symposium. Hillsdale, N.J.: Erlbaum.

SLOMAN, A. (1971). Interactions between philosophy and AI: The role of intuition and non-logical reasoning in intelligence. Artificial intelligence, 2, 209-225.

SNODGRASS, J.G. (1984). Concepts and their surface representations. Journal of Verbal Learning and Verbal Behavior, 23, 3-22.

TULVING, E. (1981). Synergistic ecphory in recall and recognition. Canadian Joumal of Psychology, $36,130-147$.

Tulving, E. (1984). Elements of episodic memory. Oxford: Clarendon Press.

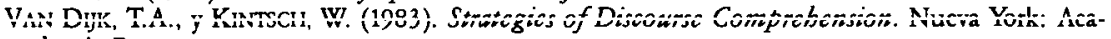
demic Press.

Wiliams, D.M., y Holland, J.D. (1981). The process of retrieval from very long-term memory. Cognitive Science, 5, 87-119. 